\title{
Juglone potentiates BRAF inhibitor-induced apoptosis in melanoma through reactive oxygen species and the p38-p53 pathway
}

\author{
ZHENG LI* ${ }^{*}$ XIAO LIU* , MING LI, JINGXIU CHAI, SHAN HE, JINFENG WU and JINHUA XU \\ Department of Dermatology, Huashan Hospital, Fudan University, Shanghai 200040, P.R. China
}

Received July 15, 2019; Accepted March 2, 2020

DOI: $10.3892 / \mathrm{mmr} .2020 .11095$

\begin{abstract}
BRAF inhibitors are some of the most effective drugs against melanoma; however, their clinical application is largely limited by drug resistance. Juglone, isolated from walnut trees, has demonstrated anti-tumour activity. In the present study, it was investigated whether juglone could enhance the responses to a BRAF inhibitor in melanoma cells (A375R and SK-MEL-5R) with an acquired resistance. These cells were treated with juglone alone, BRAF inhibitor (PLX4032) alone, or juglone combined with PLX4032. It was demonstrated that the combination of juglone and PLX4032 had synergistic effects on BRAF inhibitor-resistant melanoma cells. Juglone potentiated PLX4032-induced cytotoxicity and mitochondrial apoptosis in both A375R and SK-MEL-5R cells, which was accompanied by a decline in mitochondrial membrane potential and a decrease in $\mathrm{Bcl}-2 / \mathrm{Bax}$ ratio. Moreover, juglone combined with PLX4032 markedly increased the intracellular level of reactive oxygen species (ROS) and activated p38 and p53, as compared with juglone alone or PLX4032 alone. Pre-treatment with $\mathrm{N}$-acetyl-L-cysteine, a ROS scavenger, completely reversed the cytotoxicity induced by juglone combined with PLX4032. In conclusion, juglone potentiated BRAF inhibitor-induced apoptosis in resistant melanoma cells, and these effects occurred partially through ROS and the p38-p53 pathway, suggesting the potential of juglone as a sensitizer to BRAF inhibitors in the treatment of melanoma.
\end{abstract}

Correspondence to: Dr Jinhua Xu or Dr Jinfeng Wu, Department of Dermatology, Huashan Hospital, Fudan University, 12 Middle Urumqi Road, Shanghai 200040, P.R. China

E-mail: xjhhsyy@163.com

E-mail: wujinfeng21@163.com

*Contributed equally

Key words: juglone, BRAF inhibitor resistance, melanoma, reactive oxygen species, p38

\section{Introduction}

Melanoma is one of the most common skin cancers, but its prognosis after metastasis remains poor (1). As reported in a previous cohort study, the morbidity of melanoma in the United States, United Kingdom and Sweden will continue to increase until at least 2022 (2). Traditional treatments, including radiotherapy and chemotherapy, have low efficacy in advanced melanoma patients (3). In contrast, the discovery of BRAF inhibitors developed for BRAF gene mutations in melanoma, such as vemurafenib and dabrafenib, has largely extended progression-free-survival (PFS) compared with the PFS seen with chemotherapy. However, melanoma has been found to be highly susceptible to acquired BRAF inhibitor resistance $(4,5)$. Thus, it is imperative to explore methods that overcome resistance to BRAF inhibitors. The progress obtained in recent years reveals that the mechanisms underlying resistance to BRAF inhibitors are multiple, complicated and unpredictable (6). To date, a combination of mitogen-activated protein kinase kinase inhibitors and BRAF inhibitors has been shown to be clinically effective (7). However, the combination of these two targeted drugs can only partially impede BRAF inhibitor resistance (8). Therefore, the development of novel agents is urgently required to overcome BRAF inhibitor resistance.

Modern drug discovery programmes are gradually placing more focus on the screening of plants or other natural products, given their ease of availability, cost-effective nature and safer toxicity profiles. Juglone (5-hydroxy-1,4-naphthoquinone) is a natural naphthoquinone that is isolated from the roots, leaves, woods and fruits of walnut trees. It is not only a drug used to treat infections, but also an anti-tumour agent $(9,10)$. It has been reported that juglone exerts cytotoxic and genotoxic effects against B16F10 melanoma cells (11). It has also displayed radiation-sensitizing potential (12). Our previous study demonstrated that juglone potentiated tumour necrosis factor-related apoptosis-inducing ligand-induced apoptosis in human melanoma cells (13). The cytotoxic and sensitizing effects of juglone can be attributed to mechanisms such as the induction of reactive oxygen species (ROS) $(11,13,14)$. It has been documented that inducing ROS production might reverse the resistance to BRAF inhibition (15). Therefore, it was hypothesized that juglone might eradicate melanoma resistance to BRAF inhibitors through the induction of ROS. 
In the present study, the effect of juglone on BRAF-induced cytotoxicity was examined, and it was demonstrated that the underlying mechanisms were associated with ROS and the p38-p53 pathway.

\section{Materials and methods}

Chemicals and reagents. BRAF inhibitor PLX4032 was purchased from Selleck Chemicals, and juglone was purchased from Sigma-Aldrich; Merck KGaA. They were each dissolved in dimethyl sulfoxide (DMSO), and the final concentration of DMSO for cell culture was $<0.1 \%(\mathrm{v} / \mathrm{v})$. DMSO, 3-(4',5'-dimethylthiazol-2'-yl)-2,5-diphenyl-tetrazolium bromide (MTT) and N-acetyl-L-cysteine (NAC) were obtained from Sigma-Aldrich; Merck KGaA. 2',7'-dichlorofluorescein diacetate (DCFH-DA), 4\% paraformaldehyde, 5-ethynyl-29-deoxyuridine (EdU) cell proliferation kit with Alexa Fluor ${ }^{\circledR} 488$, and 5,5',6,6'-tetrachloro-1,1',3,3'-tetraethyl-midacarbocyanine iodide (JC-1) staining kit were purchased from Beyotime Institute of Biotechnology. JNK inhibitor (SP600125) and p38 inhibitor (SB203580) were obtained from MedChemExpress. The Annexin-V-phycoerythrin (PE) Apoptosis Detection kit (cat. c559763) was supplied by BD Biosciences. The antibodies against $\beta$-actin (cat. no. ab8226), poly(ADP-ribose) polymerase (PARP; cat. no. ab191217), survivin (cat. no. ab76424), Bcl-2 (cat. no. ab32124), Bax (cat. no. ab32503), phosphorylated (P)-p38 (cat. no. ab195049), p38 (cat. no. ab170099), P-p53 (cat. no. ab33889) and p53 (cat. no. ab179477) were obtained from Abcam, and the antibody against cytochrome $c$ (cat. no. 4280) was purchased from Cell Signaling Technology, Inc.

Cell lines and culture. Human melanoma SK-MEL-5 and A375 cell lines were obtained from American Type Culture Collection and maintained in DMEM (Invitrogen; Thermo Fisher Scientific, Inc.) containing $4 \mathrm{mM}$ L-glutamine, $3.7 \mathrm{~g} / \mathrm{l}$ sodium bicarbonate, $4.5 \mathrm{~g} / \mathrm{l}$ glucose and $10 \%$ foetal bovine serum (Invitrogen; Thermo Fisher Scientific, Inc.). The cells were maintained in a $5 \% \mathrm{CO}_{2}$ humidified incubator at $37^{\circ} \mathrm{C}$. Both SK-MEL-5 and A375 cells carrying the BRAFV600E mutation are sensitive to vemurafenib treatment $(16,17)$. A SK-MEL-5 subline with acquired resistance (SK-MEL-5R) to PLX4032 was generated by continuous exposure of parental SK-MEL-5 cells to gradually increasing concentrations of PLX4032 (from $0.1 \mu \mathrm{M}$ up to $1 \mu \mathrm{M}$ ) over a period of 3 months. The fold resistance was intermittently evaluated by cell viability assays and it was found that SK-MEL-5R showed higher cell viability compared with SK-MEL-5 when treated with different concentrations of PLX4032 (data not shown). A375 cells with acquired resistant to PLX4032 (A375R) were generated by the same method, with the concentration of PLX4032 increasing from $0.1 \mu \mathrm{M}$ up to $2 \mu \mathrm{M}$, as previously described (18).

Cell viability assays. The cytotoxic effects of juglone and/or PLX4032 on SK-MEL-5R and A375R cells were assessed by MTT assays. Briefly, 4,000 cells in $200 \mu \mathrm{l}$ cell culture medium/well were seeded into 96 -well plates and incubated overnight at $37^{\circ} \mathrm{C}$. After NAC (0 and $\left.2 \mathrm{mM}\right)$, SB203580 (0 and $10 \mu \mathrm{M})$ or SP600125 (0 and $10 \mu \mathrm{M})$ pre-treatment for $1 \mathrm{~h}$ at $37^{\circ} \mathrm{C}$, the cells were treated with juglone $(0,5$ or $7.5 \mu \mathrm{M})$ and/or PLX4032 $(0,5$ or $10 \mu \mathrm{M})$ for $24 \mathrm{~h}$ at $37^{\circ} \mathrm{C}$. DMEM containing corresponding amounts of $0.1 \%$ DMSO were used as vehicle controls. Then, $20 \mu \mathrm{l}$ MTT solution $(5 \mathrm{mg} / \mathrm{ml})$ was added to each well, and the cells were incubated for $2 \mathrm{~h}$ at $37^{\circ} \mathrm{C}$. Subsequently, the formazan crystals that formed were dissolved with $100 \mu \mathrm{l}$ DMSO, and the optical density (OD) was measured at $570 \mathrm{~nm}$ on a microplate spectrophotometer (Infinite 200 PRO; Tecan Group, Ltd.). The cell viability was determined using the following formula: Cytotoxicity $(\%)=(\mathrm{OD}$ treatment/OD vehicle control $) \times 100$. For crystal violet staining, $2 \times 10^{5}$ cells/well were seeded into 6 -well plates, followed by exposure to juglone $(0$ and $5 \mu \mathrm{M})$ and/or PLX4032 $(0$ and $10 \mu \mathrm{M})$ for $72 \mathrm{~h}$ at $37^{\circ} \mathrm{C}$. Then, the cells were fixed with $10 \%$ formalin for $10 \mathrm{~min}$ at room temperature and stained with $0.05 \%$ crystal violet solution (CV) in distilled water for $30 \mathrm{~min}$ at room temperature. Finally, the $\mathrm{CV}$ was removed, cells were washed twice with distilled water and images were captured using a Flatbed Scanner (Canon LiDE 220; Canon Inc.).

Cell proliferation assays. To assess the cell proliferation, an EdU assay kit was used according to the manufacturer's instructions. Briefly, $2 \times 10^{5}$ cells/well were seeded in a 6 -well plate and treated with juglone $(0$ and $5 \mu \mathrm{M})$ and/or PLX4032 (0 and $10 \mu \mathrm{M}$ ) for $24 \mathrm{~h}$, followed by incubation with cell culture medium containing $10 \mu \mathrm{M} \mathrm{EdU}$ at $37^{\circ} \mathrm{C}$ for $2 \mathrm{~h}$. Then, the cells were fixed with $4 \%$ paraformaldehyde for $15 \mathrm{~min}$ and permeabilized with $0.3 \%$ Triton X-100 in PBS for 15 min. After washing with PBS, cells were incubated with click additive solution (supplied with the kit) for $30 \mathrm{~min}$ in the dark at room temperature. Finally, cells were washed with $0.3 \%$ Triton X-100 and incubated with Hoechst 33342 for $10 \mathrm{~min}$ at room temperature. After washing with PBS 3 times for 5 min each time, the images were captured using a fluorescence microscope (Axio Vert. A1; Zeiss GmbH) at 400x magnification and the subsequent images were analyzed using ImageJ software (v1.52 g; National Institutes of Health). The data comprised three replicates.

Apoptosis assays. Apoptosis was measured using flow cytometry. Cells were seeded into 6-well culture plates at a density of $2 \times 10^{5}$ cells/well, and then treated with juglone $(0$ and $5 \mu \mathrm{M})$ and/or PLX4032 (0 and $10 \mu \mathrm{M})$ for $24 \mathrm{~h}$ at $37^{\circ} \mathrm{C}$. Next, cells were harvested, washed with PBS twice and resuspended in $1 \mathrm{X}$ binding buffer (supplied with kit). Finally, the cells were incubated with Annexin V-PE and 7-AAD in the dark at room temperature for $15 \mathrm{~min}$, and analysed using a flow cytometer (Attune NxT; Thermo Fisher Scientific, Inc.). The data were analysed using FlowJo software V6.0 (Tree Star, Inc.).

Western blot analysis. Cells were harvested following the pre-treatment with NAC (0 and $2 \mathrm{mM})$, SB203580 (0 and $10 \mu \mathrm{M})$ or SP600125 $(0$ and $10 \mu \mathrm{M})$ for $1 \mathrm{~h}$ at $37^{\circ} \mathrm{C}$ and treated with juglone ( 0 and $5 \mu \mathrm{M})$ and/or PLX4032 $(0$ and $10 \mu \mathrm{M})$ for $6 \mathrm{~h}$ at $37^{\circ} \mathrm{C}$. The cells were then lysed in RIPA buffer (Beyotime Institute of Biotechnology). The mixture was centrifuged at $12,000 \mathrm{x} \mathrm{g}$ at $4^{\circ} \mathrm{C}$ for $15 \mathrm{~min}$, and the supernatants were collected. The protein concentrations were determined using a bicinchoninic acid protein assay kit (Bio-Rad Laboratories, Inc.). Next, $30 \mu \mathrm{g}$ cellular proteins were separated on a 10 or $12 \%$ SDS-polyacrylamide gel and electroblotted onto a PVDF membrane. Membranes were blocked with 5\% 
milk in Tween-20/Tris-buffered saline (TBST) for $1 \mathrm{~h}$ at room temperature, prior to incubation overnight at $4^{\circ} \mathrm{C}$ with 1:1,000 dilution of primary antibodies against $\beta$-actin, PARP, survivin, Bcl-2, Bax, p-p38, p38, p-p53, p53, JNK, p-JNK and cytochrome $c$. Membranes were washed with TBST, followed by $1 \mathrm{~h}$ incubation at room temperature in 1:4,000 dilution of horseradish peroxidise-conjugated secondary antibodies (cat. nos. 7074 and 7076; Cell Signaling Technology, Inc.). After washing again, the blots were developed using Supersignal West Femto Chemiluminescent substrate (Thermo Fisher Scientific, Inc.). Images were acquired by the ChemiDoc ${ }^{\mathrm{TM}}$ MP Imaging system (Bio-Rad Laboratories, Inc.). The band intensities were semi-quantified using ImageJ software and normalized to $\beta$-actin as the loading control. The western blot assays were repeated three times.

Evaluation of ROS. ROS were detected using the fluorescent probe DCFH-DA. Briefly, the cells were seeded into 6-well culture plates at a density of $2 \times 10^{5}$ cells/well, and then treated with juglone $(0$ and $5 \mu \mathrm{M})$ and/or PLX4032 (0 and $10 \mu \mathrm{M})$ for $2 \mathrm{~h}$. The cells were then washed with PBS, incubated in serum-free medium, and loaded with DCFH-DA $(10 \mu \mathrm{M})$. Following incubation in the dark at $37^{\circ} \mathrm{C}$ for $30 \mathrm{~min}$, the cells were washed with PBS and vortexed in $700 \mu 1$ lysis buffer (90\% DMSO:10\% PBS)/well for $15 \mathrm{~min}$. The cell lysis buffer was transferred into a black 96-well Immuno Plate (Thermo Fisher Scientific, Inc.), and fluorescence was detected using a fluorescence microplate reader (Infinite 200 PRO; Tecan Group, Ltd.) at an excitation wavelength of $485 \mathrm{~nm}$, and an emission wavelength of $535 \mathrm{~nm}$.

Analysis of mitochondrial membrane potential $\left(\Delta \Psi_{M}\right) . \Delta \Psi_{\mathrm{M}}$ was evaluated using a JC-1 kit. Briefly, $1 \times 10^{6}$ cells seeded in a 6-well plate were cultured with juglone $(0$ and $5 \mu \mathrm{M})$ and/or PLX4032 ( 0 and $10 \mu \mathrm{M})$ for $3 \mathrm{~h}$. Then, the cells were labelled with $\mathrm{JC}-1$ and incubated for $20 \mathrm{~min}$ at $37^{\circ} \mathrm{C}$, according to the manufacturer's instructions. Cells were washed twice, resuspended and detected by flow cytometer (Attune NxT; Thermo Fisher Scientific, Inc.); the PE and FITC detection channels were selected at emission wavelengths of 530 and $585 \mathrm{~nm}$, and an excitation wavelength of $488 \mathrm{~nm}$. The data were analysed by FlowJo software V6.0 (Tree Star, Inc.). Mitochondrial carbonyl cyanide 3-chlorophenylhydrazone (supplied in kit) was used as a positive control. The percentage of depolarized mitochondrial was calculated as previously described (19).

Statistical analysis. All results are presented as the mean \pm standard deviation of at least three independent experiments. Statistical analysis was performed by SPSS Statistics 21.0 software (IBM Corp.). Student's t-test was used for comparisons between two groups, and one-way ANOVA and subsequent Tukey's post-hoc analysis was applied for comparison of more than two independent groups. $\mathrm{P}<0.05$ was considered to indicate a statistically significant difference.

\section{Results}

Juglone sensitizes melanoma cells to PLX4032-induced cytotoxicity. MTT was used to determine the cytotoxic effects of juglone and/or PLX4032 on melanoma cells. As shown in Fig. 1A and C, the combination of juglone and PLX4032 in SK-MEL-5R and A375R melanoma cells induced a much higher cytotoxicity than juglone or PLX4032 alone $(\mathrm{P}<0.01)$. In addition, according to the MTT results, the combination index (CI) of juglone and PLX4032 cotreatment in SK-MEL-5R cells was determined using the Chou-Talalay method (20). The CI values varied from $0.34-0.65$, which suggested a strong synergistic effect between juglone and PLX4032 (Fig. 1B). Crystal violet staining confirmed that the combination of juglone and PLX4032 further induced cytotoxicity to melanoma cells, compared with either juglone alone or PLX4032 alone (Fig. 1D). EdU assays also suggested that cell proliferation was further inhibited after treatment with a combination of PLX4032 and juglone (Fig. 1E and F).

Juglone potentiates PLX4032-induced apoptosis in melanoma. To further demonstrate the synergistic effect of juglone and PLX4032, flow cytometry analysis was performed to measure the percentages of both Annexin $\mathrm{V}^{+} / 7-\mathrm{AAD}^{-}$(early apoptotic cells) and Annexin $\mathrm{V}^{+} / 7-\mathrm{AAD}^{+}$(late apoptotic/necrotic cells) cells. In SK-MEL-5R cells, PLX4032 alone induced only minor apoptosis. However, the combination of juglone and PLX4032 exhibited a much higher rate of apoptosis than either PLX4032 or juglone alone (Fig. 2A and C; $<<0.01$ ). Similar results were observed in A375R cells (Fig. $2 \mathrm{~B}$ and $\mathrm{C}$; $\mathrm{P}<0.01$ ).

PARP is one of the terminal pro-apoptotic proteins. The cleaved forms of PARP are its active forms (21). Survivin, on the other hand, inhibits apoptosis $(22,23)$. As demonstrated by western blotting, the protein expression of cleaved PARP in the juglone and PLX4032 combination group was the highest, while the protein expression of survivin was the lowest, as compared with levels in either the PLX4032 or juglone alone groups (Fig. 2D-F).

Juglone enhances PLX4032-induced apoptosis through the mitochondrial pathway. $\Delta \Psi_{\mathrm{M}}$ decline is one of the earliest indicators of intrinsic apoptosis. In the present study, the fluorescent probe JC-1 was used to determine the effect of juglone on $\Delta \Psi_{M}$ in SK-MEL-5R cells. When the mitochondrial potential is normal, JC-1 forms a polymer in the mitochondrial matrix and exhibits red fluorescence with an emission of $590 \mathrm{~nm}$; however, when the $\Delta \Psi_{\mathrm{M}}$ is downregulated, JC-1 changes into a monomeric form that yields green fluorescence with an emission of $530 \mathrm{~nm}$, so the PE and FITC channel were selected to measure the fluorescence intensity of JC-1 dye in the cells (24). As shown in Fig. 3A and B, juglone caused a marked decrease in $\Delta \Psi_{\mathrm{M}}$ compared with the vehicle control. Moreover, the combination of juglone and PLX4032 further decreased the $\Delta \Psi_{\mathrm{M}}$, and the percentage of cells with low potential was increased from $44.5 \%$ in the juglone alone group to $55.8 \%$ when cotreated with juglone and PLX4032.

The pro-apoptotic protein Bax and anti-apoptotic protein Bcl-2 serve central roles in the mitochondria-dependent apoptotic pathway; Bax can be translocated to the outer mitochondrial membrane following a death signal, where it promotes a permeabilization that favors the release of different apoptogenic factors, such as cytochrome $c$ (25). In addition, the ability of Bax to form channels on the mitochondrial membrane can be inhibited by Bcl-2 (26). As demonstrated by western blot analysis (Fig. 3C), the protein expression of Bax was notably 

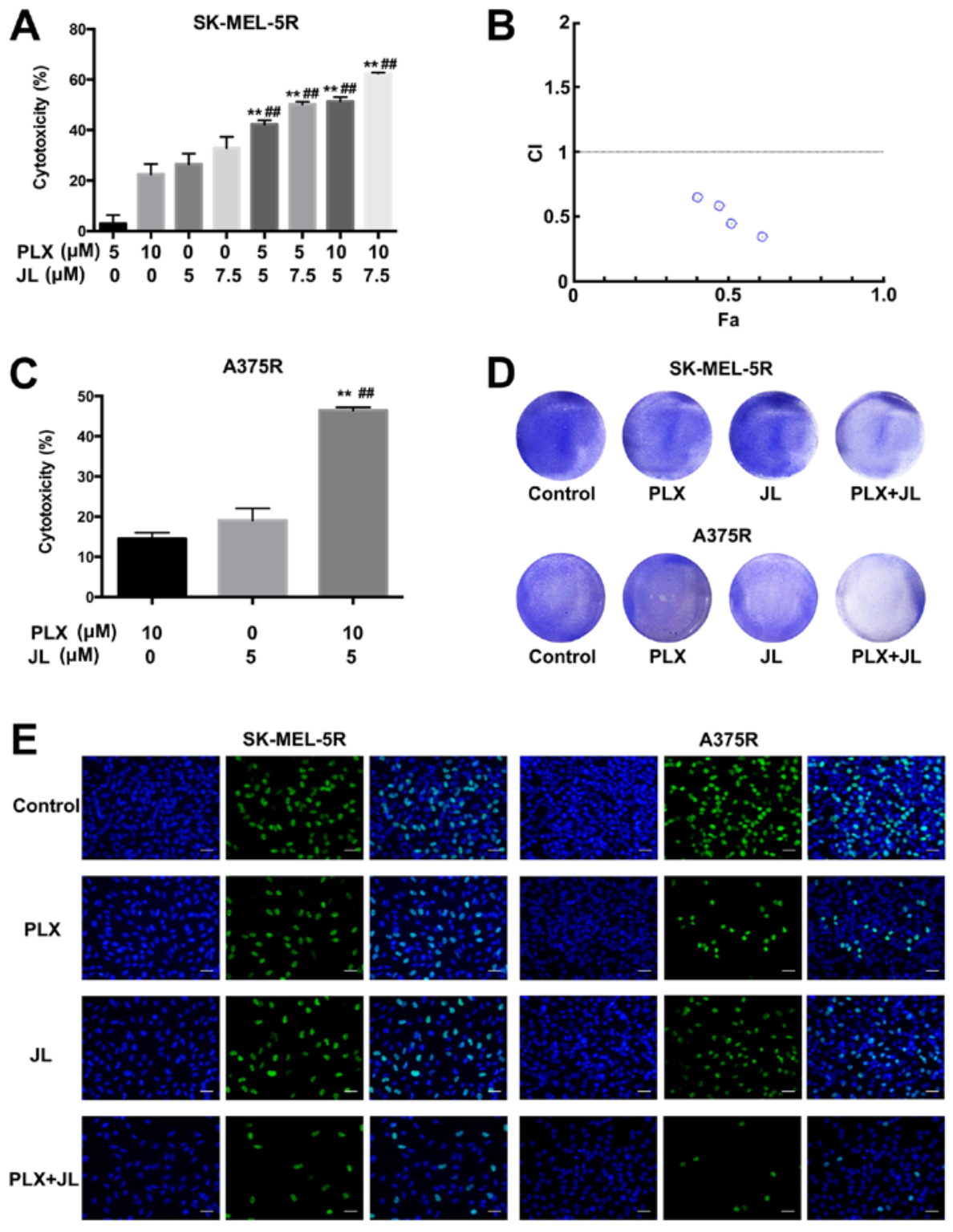

Hoechst

EdU

Merge

Hoechst

EdU

Merge
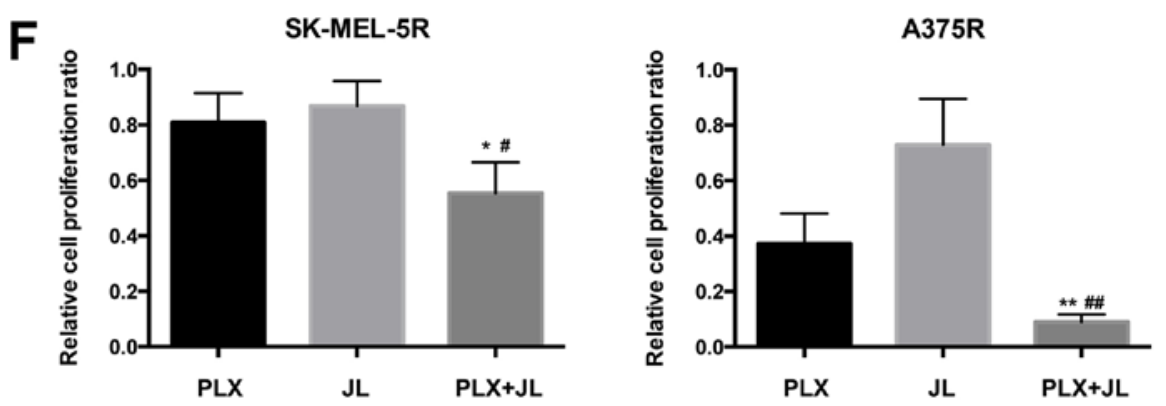

Figure 1. Juglone sensitizes melanoma cells to PLX4032-induced cytotoxicity. (A) MTT assay of SK-MEL-5R cells; SK-MEL-5R cells were treated with juglone $(0,5$ and $7.5 \mu \mathrm{M})$ and/or PLX4032 $(0,5$ and $10 \mu \mathrm{M})$ for $24 \mathrm{~h} .{ }^{* * *} \mathrm{P}<0.01$ vs. same concentration PLX alone, ${ }^{\text {\# }} \mathrm{P}<0.01$ vs. same concentration JL alone. (B) CI of juglone and PLX4032. (C) MTT assay of A375R cells; A375R cells were treated with juglone $(0$ and $5 \mu \mathrm{M})$ and/or PLX4032 $(0$ and $10 \mu \mathrm{M})$ for $48 \mathrm{~h} .{ }^{* *} \mathrm{P}<0.01$ vs. PLX, ${ }^{\# \#} \mathrm{P}<0.01$ vs. JL. (D) Crystal violet staining; SK-MEL-5R and A375R cells were exposed to juglone (0 and $\left.5 \mu \mathrm{M}\right)$ and/or PLX4032 $(0$ and $10 \mu \mathrm{M})$ for $72 \mathrm{~h}$. The cells were then fixed and stained with crystal violet solution. (E) EdU assay; cells were double-stained with EdU (green) and Hoechst 33342 (blue). Scale bar, $20 \mu \mathrm{m}$. (F) Relative cell proliferation rates were analyzed according to the EdU assay results. ${ }^{* *} \mathrm{P}<0.01$, ${ }^{*} \mathrm{P}<0.05$ vs. PLX; ${ }^{\# \#} \mathrm{P}<0.01,{ }^{\#} \mathrm{P}<0.05$ vs. JL. CI, combination index; EdU, 5-ethynyl-29-deoxyuridine; JL, juglone; PLX, PLX4032.

increased after cotreatment with juglone and PLX4032, while no changes were seen in the juglone or PLX4032 only groups. $\mathrm{A}$ reduction in the $\mathrm{Bcl}-2 / \mathrm{Bax}$ ratio was observed after the combination drug treatment in SK-MEL-5R cells (Fig. 3D). The combination of PLX4032 and juglone also increased the protein expression of cytochrome $c$ (Fig. 3C and E). 
A

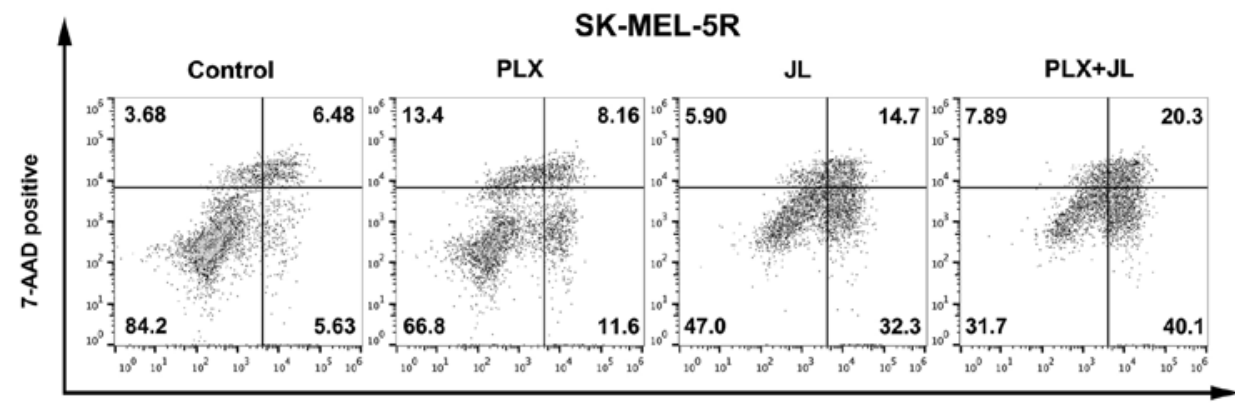

Annexin V positive

B

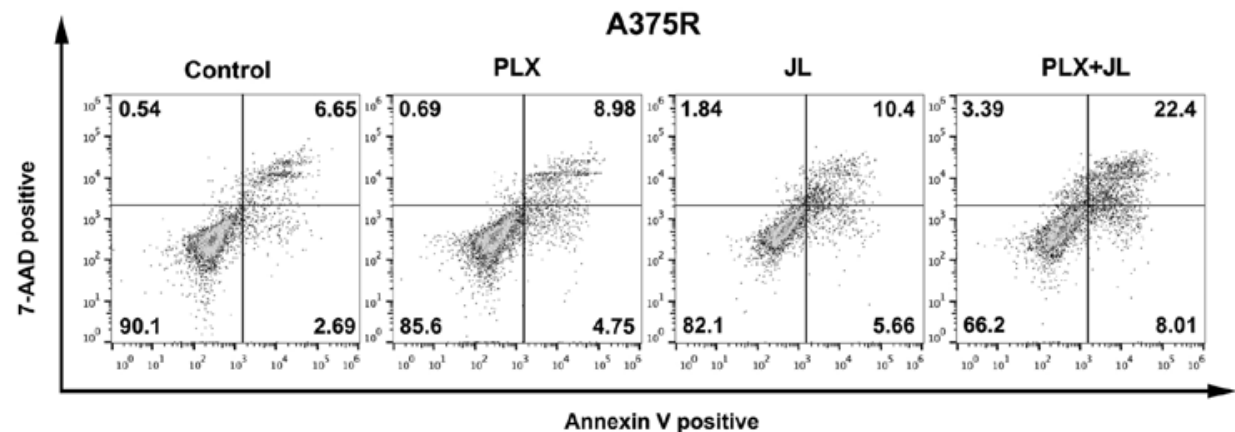

C
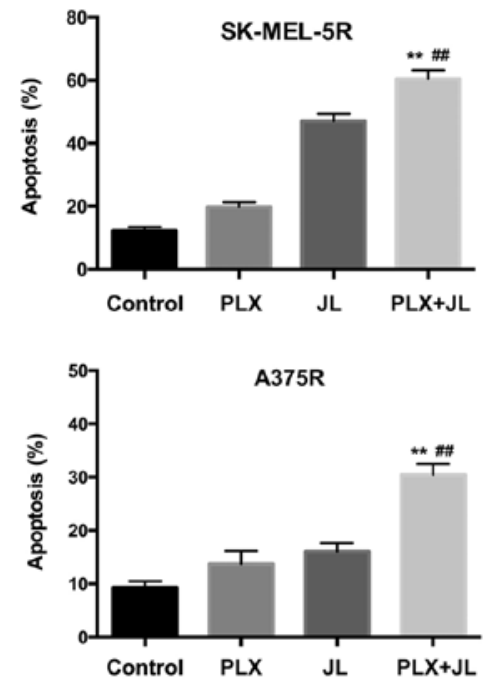

D SK-MEL-5R

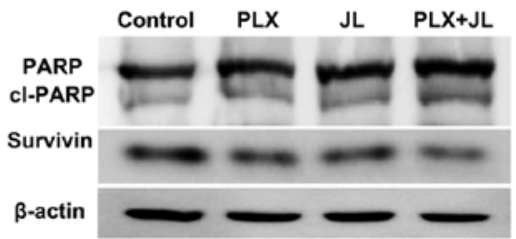

A375R
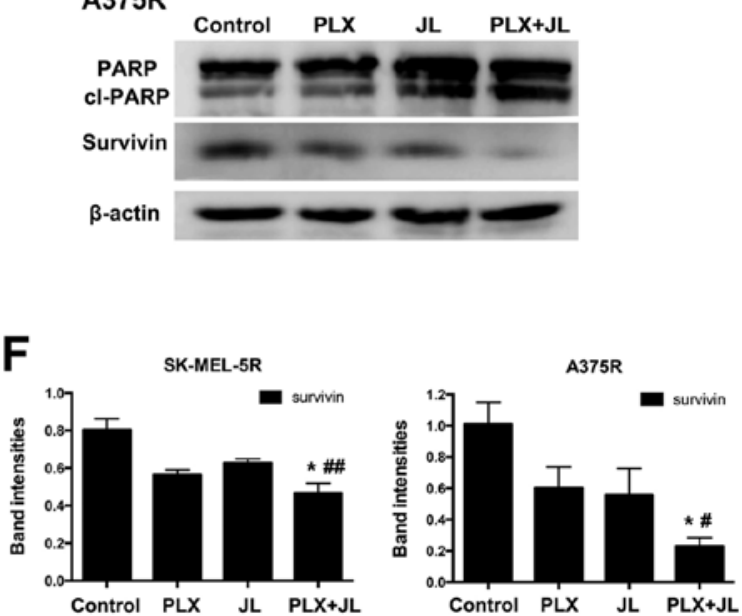

Figure 2. Juglone potentiates PLX4032-induced melanoma cell apoptosis. Flow cytometric analysis of (A) SK-MEL-5R cell apoptosis and (B) A375R cell apoptosis. (C) Quantitative presentation of early and late apoptotic cells. Cells were treated with juglone ( 0 and $5 \mu \mathrm{M})$ and/or PLX4032 ( 0 and $10 \mu \mathrm{M})$ for $24 \mathrm{~h}$. Next, cells were stained with Annexin V and 7-AAD, followed by analysis in a flow cytometer. Experiments were repeated three times. ${ }^{* *} \mathrm{P}<0.01 \mathrm{vs.} \mathrm{PLX}$ alone; ${ }^{\# \#} \mathrm{P}<0.01$ vs. JL alone. (D) Representative western blot images of PARP and survivin; SK-MEL-5R and A375R cells were treated with juglone $(0$ and $5 \mu \mathrm{M})$ and/or PLX4032 ( 0 and $10 \mu \mathrm{M})$ for $6 \mathrm{~h}$. Semi-quantification of band intensities of (E) cl-PARP and (F) surviving in SK-MEL-5R and A3755R cells. "P<0.05, ${ }^{* *} \mathrm{P}<0.01$ vs. $\mathrm{PLX} ;{ }^{\#} \mathrm{P}<0.05,{ }^{\# \#} \mathrm{P}<0.01$ vs. JL. JL, juglone; PARP, poly(ADP-ribose) polymerase; $\mathrm{Cl}-$, cleaved.

Juglone increases the cellular ROS level and activated p38, p53 and JNK in SK-MEL-5R cells. Since mitochondrial apoptosis is associated with the generation of ROS (27), the production of intracellular ROS was examined using the oxidant-sensitive fluorescent probe DCFH-DA. The amount of ROS was analysed in cells treated with juglone and/or PLX4032 using a fluorescence microplate reader. Both PLX4032 or juglone alone increased the level of ROS. However, the level of ROS was significantly higher in the juglone and PLX4032 cotreatment group compared with the juglone or PLX4032 alone groups (Fig. 4A). 

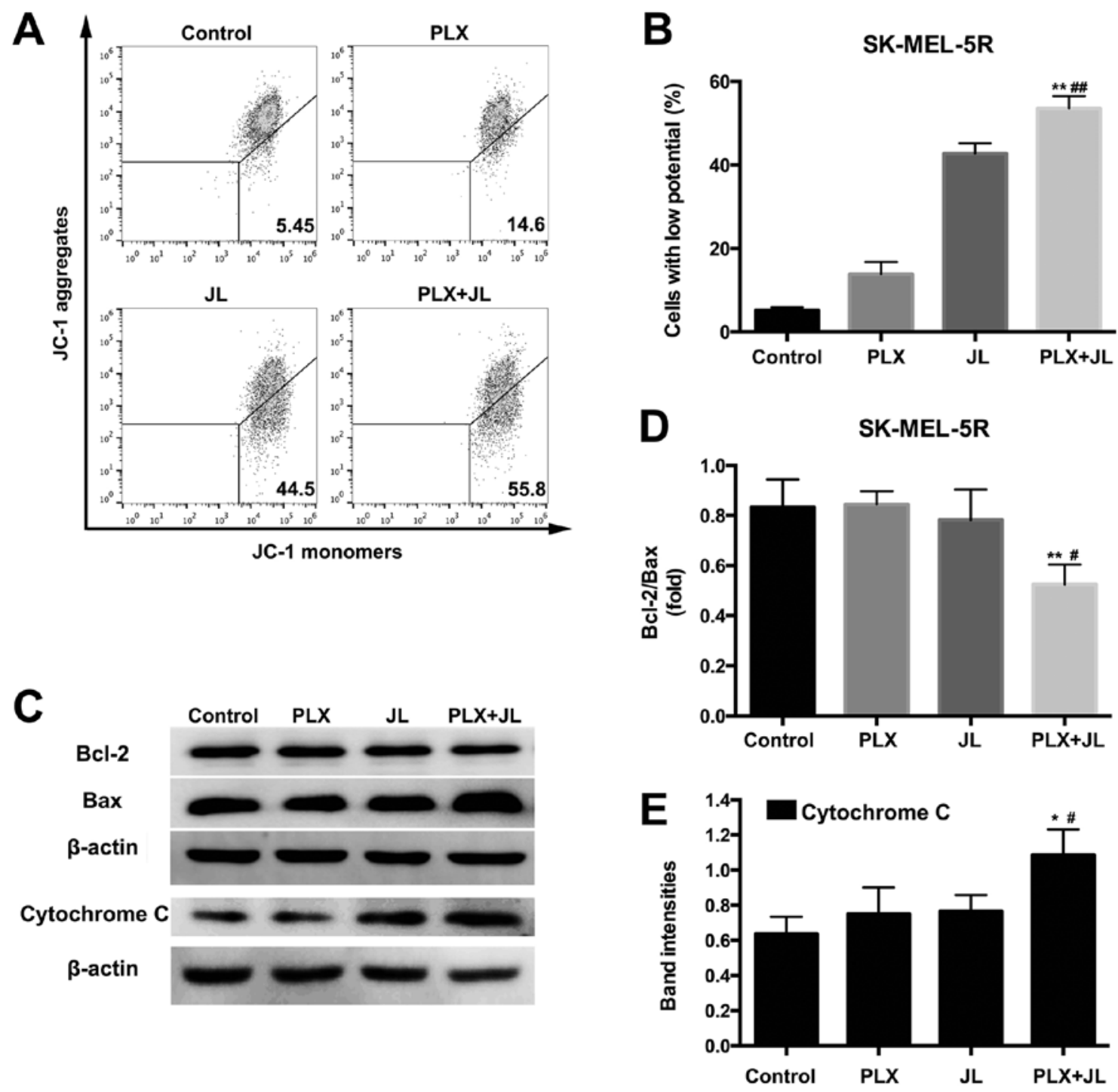

Figure 3. Juglone enhances PLX4032-induced mitochondrial apoptosis. (A) Mitochondrial membrane potential analysed by JC-1 fluorescence with flow cytometry. (B) Quantification of cells with low potential. ${ }^{* *} \mathrm{P}<0.01$ vs. PLX alone; ${ }^{\# \#} \mathrm{P}<0.01$ vs. JL alone. (C) Representative western blot images of Bcl-2, Bax and cytochrome $c$. (D) Bcl-2/Bax band intensity ratio. ${ }^{* *} \mathrm{P}<0.01$ vs. PLX alone; ${ }^{*} \mathrm{P}<0.05$ vs. JL alone. (E) Semi-quantification of band intensities of cytochrome $c$. ${ }^{*} \mathrm{P}<0.05$ vs. PLX; ${ }^{\prime} \mathrm{P}<0.05$ vs. JL. JC-1, 5,5',6,6'-etrachloro-1,1',3,3'-tetraethyl-imidacarbocyanine iodide; PLX, PLX4032; JL, juglone.

Previous evidence has indicated that ROS can also mediate cell death via key modulators, such as p38 mitogen-activated protein kinase (MAPK) and p53 $(28,29)$. To test whether the p38-p53 pathway was activated by juglone and PLX4032 cotreatment, western blotting was used to measure the protein expression of p38, p53 and their phosphorylated counterparts. As demonstrated in Fig. 4B, in SK-MEL-5R cells, juglone and PLX4032 cotreatment markedly increased p38 and p53 phosphorylation compared with treatment of PLX4032 or juglone alone. JNK (Thr183/Tyr185) activation was also observed after juglone and PLX4032 cotreatment.

NAC and 38 inhibitor pre-treatment partially reverse juglone and PLX4032 cotreatment-induced cytotoxicity. After cells were pre-treated with NAC (a ROS scavenger), or SB203580 (a p38 inhibitor), juglone and PLX4032 cotreatment-induced cytotoxicity was partially reversed (Fig. 4C and D). Furthermore, as demonstrated by western blotting, the protein expression of cleaved PARP in the NAC or p38 inhibitor pre-treatment group was lower than the protein expression in the juglone/PLX4032 combination group without NAC or p38 inhibitor pre-treatment (Fig. 4E-H; $\mathrm{P}<0.05$ ). However, pre-treatment with SP600125 (a JNK inhibitor), failed to reverse the cytotoxicity induced by juglone and PLX4032 cotreatment (Fig. 4D). This was consistent with the inability of JNK inhibitor to reduce the levels of cl-PARP (Fig. 4F and H). These results suggested that ROS production and $\mathrm{p} 38$ activation play important roles in juglone-induced PLX4032 sensitization.

\section{Discussion}

It has been reported that juglone displays anti-tumour activities in a variety of tumour cell lines $(11,14,30)$, and that treatment of B16F10 melanoma cells with juglone results in a concentration-dependent reduction in cell viability. For example, after $24 \mathrm{~h}$ exposure to $5 \mu \mathrm{M}$ juglone, cytotoxicity was increased by only $\sim 20 \%$ compared with the vehicle control (11). This 
A

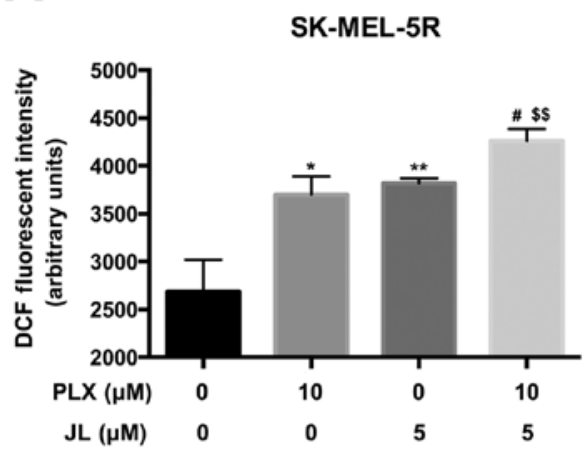

B

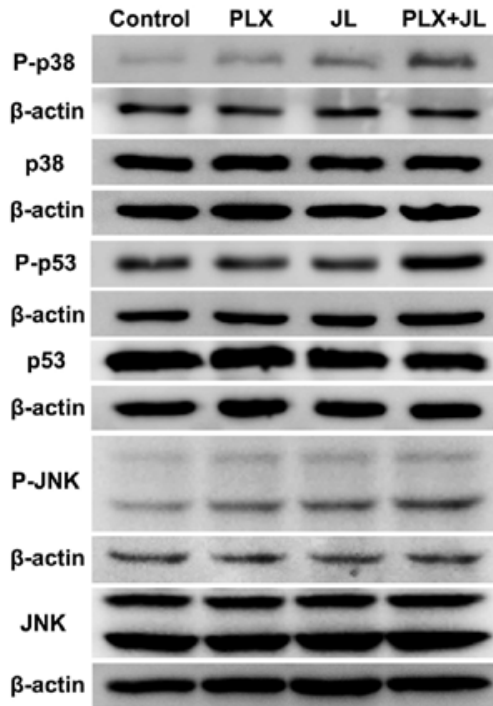

C

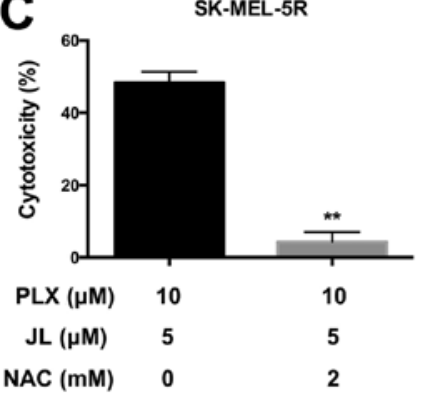

D

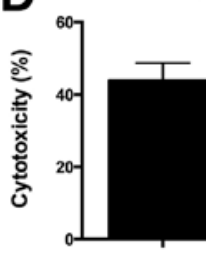

$\operatorname{PLX}(\mu \mathrm{M}) \quad 10$

$J L(\mu M)$

p38i $(\mu \mathrm{M})$
SK-MEL-5R

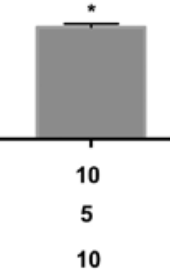

SK-MEL-5R

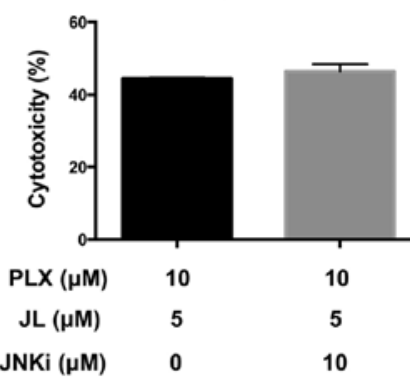

$\mathbf{E}$

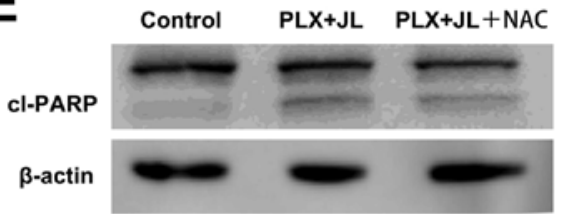

G

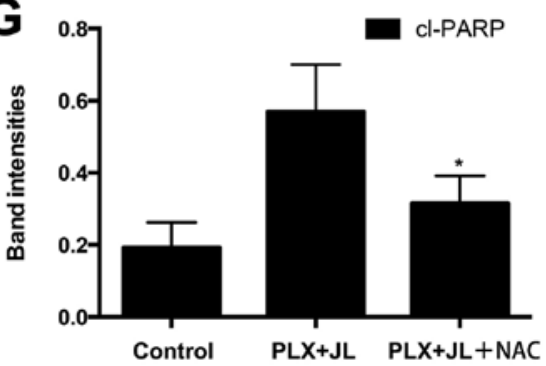

$\mathbf{F}$

$P L X+J L \quad P L X+J L+J N K i \quad P L X+J L+p 38 i$

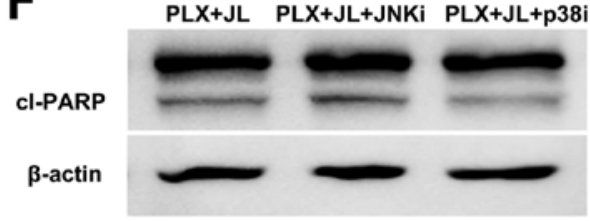

H

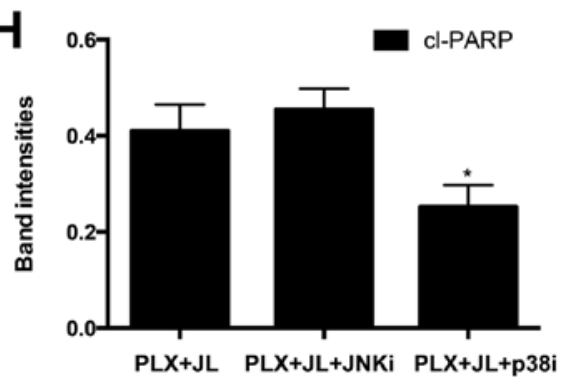

Figure 4. Juglone in combination with PLX4032 increases the production of ROS and activates p38, p53 and JNK. (A) Intracellular ROS levels measured by DCFH-DA. SK-MEL-5R cells were treated with juglone $(0$ and $5 \mu \mathrm{M})$ and/or PLX4032 $(0$ and $10 \mu \mathrm{M})$ for $2 \mathrm{~h}$, fluorescence intensity was monitored by a fluorescence microplate reader. ${ }^{* *} \mathrm{P}<0.01,{ }^{*} \mathrm{P}<0.05$ vs. control; ${ }^{*} \mathrm{P}<0.05$ vs. $\mathrm{PLX} ;{ }^{\$ \$} \mathrm{P}<0.01$, vs. JL. (B) Representative western blot images of p-p38, p38, p-p53, p53, p-JNK and JNK. (C) NAC pre-treatment partially reverses cytotoxic effect of juglone. SK-MEL-5R cells were pre-treated with NAC (0 and 2 mM) for $1 \mathrm{~h}$, followed by juglone and PLX4032 cotreatment for $24 \mathrm{~h}$. The cytotoxicity was measured using MTT assays. * P<0.01 vs. NAC non-pre-treatment. (D) p38i partially reversed the cytotoxic effect of juglone and PLX4032. SK-MEL-5R cells were pre-treated with $\mathrm{p} 38 \mathrm{i}(0$ and $10 \mu \mathrm{M})$ or JNKi $(0$ and $10 \mu \mathrm{M})$ for $1 \mathrm{~h}$, followed by juglone and PLX4032 cotreatment for $24 \mathrm{~h}$. Cytotoxicity was measured using MTT assays. "P<0.05. (E) Representative western blot images of cl-PARP from cells pre-treated with NAC. (F) Representative western blot images of cl-PARP from cells pre-treated with p38i and JNKi. (G) Semi-quantification of band intensities of cl-PARP from part (E). (H) Semi-quantification of band intensities of cl-PARP from part (F). " $\mathrm{P}<0.05$ vs. PLX and JL cotreatment. NAC, N-acetyl-L-cysteine; PARP, poly(ADP-ribose) polymerase; DCFH-DA, 2',7'-dichlorofluorescein diacetate; DCF, 2',7'-dichlorofluorescein; JL, juglone; ROS, reactive oxygen species; P-, phosphorylated; Cl-, cleaved; p38i, p38 inhibitor; JNKi, JNK inhibitor.

is in accordance with the finding in the present study that juglone at a low concentration possesses weak activity against BRAF-mutant melanoma cells.
Juglone has displayed potent cytotoxic properties against chemo-resistant and radio-resistant tumour cell lines both in vitro and in vivo $(12,31)$. In addition, it has been considered 
as a sensitizing agent for overcoming trastuzumab resistance in human breast cancer SKBR3 cells (32). Combination therapy with a BRAF inhibitor and juglone might provide a novel strategy for overcoming BRAF inhibitor resistance. In the present study, to the best of our knowledge, it was reported for the first time that juglone enhanced the response to a BRAF inhibitor in BRAF inhibitor-resistant melanoma cells.

Apoptosis is a form of complex signalling-controlled cell death. The two main pathways of apoptosis are the extrinsic pathway and the intrinsic pathway, which is also called the mitochondrial pathway. The intrinsic pathway that initiates apoptosis involves a diverse array of non-receptor-mediated stimuli. These produce intracellular signals that cause changes in the inner mitochondrial membrane (33). The anti-cancer effects of juglone can be partially attributed to mitochondrial apoptosis $(30,34)$. Juglone has been found to markedly alter the levels of Bcl-2 and Bax, thereby releasing cytochrome $c$ into the cell cytoplasm, and inducing subsequent caspase activation and activation of PARP $(30,34)$. In the present study, it was found that juglone potentiated BRAF inhibitor-induced apoptosis in both A375R and SK-MEL-5R cells, which was accompanied by a decline in $\Delta \Psi_{\mathrm{M}}$, and a reduction of the Bcl-2/Bax ratio.

ROS are one of the stimuli that can induce the mitochondrial apoptotic pathway. An increase in ROS leads to the opening of the mitochondrial membrane permeability transition pore, which destroys the integrity of the mitochondrial membrane, inducing $\Delta \Psi_{\mathrm{M}}$ loss (33). Meanwhile, damaged mitochondria produce more ROS and accelerate apoptosis. It was revealed that the cytotoxicity of juglone is generally attributed to its ability to induce redox cycling with consequent ROS, leading to oxidative stress-mediated cell death (35). Elevated ROS levels have been observed after juglone treatment in multiple human cancer lines, including glioma, gastric cancer and melanoma cells $(14,30,36)$. In the present study, juglone alone significantly increased the levels of ROS in BRAF inhibitor-resistant melanoma cells. Moreover, the level of ROS was higher in the juglone and PLX4032 cotreatment group than in the single agent treatment groups. Pre-treatment with NAC, a ROS scavenger, partially reversed juglone and PLX4032 combination-induced cytotoxicity. Therefore, it was concluded that the synergistic effects of juglone and a BRAF inhibitor in melanoma cells were partially mediated by ROS.

In addition to disrupting the mitochondrial membrane, ROS act as secondary messengers to trigger apoptotic signals (37). The downstream molecules affected by ROS include MAPK, PI3K/AKT, phospholipase $\gamma 1$, NF- $\mathrm{B}$ and Janus kinase, among which the MAPK pathway is a complex family that transmits extracellular signals to the intracellular environment. MAPK pathways include ERK, JNK and p38. Unlike ERK, both JNK and p38 mediate tumour cell apoptosis. JNK and p38 and are also more stress-responsive than ERK, and are often termed stress-activated protein kinases (38). p53 is a direct downstream effector of p38 $(28,39)$, which causes cell cycle arrest and induces cellular senescence and apoptosis (40). In the present study, it was demonstrated that the p38-p53 pathway was activated after juglone and PLX4032 cotreatment.
A previous study has demonstrated that juglone induced HeLa cells to undergo apoptosis by activating the JNK/c-Jun pathway (41). Previously, a JNK activator was reported to exert anti-tumour activity in vemurafenib-resistant melanoma cells, and this indicated that the induction of apoptosis through the activation of the JNK pathway might represent a novel strategy to overcome resistance to vemurafenib (42). In the present study, it was demonstrated that juglone and PLX4032 cotreatment activated JNK. However, unlike p38 inhibition, inhibition of JNK could not reverse the juglone-induced enhanced sensitivity to PLX4032. Therefore, this suggested that $\mathrm{p} 38$, and not JNK, plays a crucial role in the induction of apoptosis following juglone and PLX4032 cotreatment. Juglone has been reported to possess low cytotoxicity in human peripheral blood mononuclear cells (43). However, it is unclear whether juglone combined with a BRAF inhibitor is toxic in normal cells. Further in vivo studies are necessary to determine the efficacy and safety of juglone and BRAF inhibitor cotreatment in BRAF inhibitor-resistant melanoma.

In conclusion, juglone potentiated the BRAF inhibitor-induced apoptosis in BRAF inhibitor-resistant melanoma cells, and these effects were partially mediated through ROS and the p38-p53 pathway, thereby suggesting the potential of juglone as a sensitizer to BRAF inhibitors in the treatment of melanoma.

\section{Acknowledgements}

Not applicable.

\section{Funding}

The present study was supported by grants from the National Natural Science Foundation of China (grant no. 81673917) and Shanghai Science and Technology Committee (grant no. 13JC1401401).

\section{Availability of data and materials}

The datasets used and/or analysed during the present study are available from the corresponding author on reasonable request.

\section{Authors' contributions}

JX and JW designed the research and interpreted the data; ZL and XL performed the experiments and wrote the manuscript. ML, JC and SH analyzed the data. All authors read and approved the final manuscript.

\section{Ethics approval and consent to participate}

Not applicable.

\section{Patient consent for publication}

Not applicable.

\section{Competing interests}

The authors declare that they have no competing interests. 


\section{References}

1. Siegel RL, Miller KD and Jemal A: Cancer statistics, 2019 CA Cancer J Clin 69: 7-34, 2019.

2. Whiteman DC, Green AC and Olsen CM: The growing burden of invasive melanoma: Projections of incidence rates and numbers of new cases in six susceptible populations through 2031. J Invest Dermatol 136: 1161-1171, 2016.

3. Flaherty KT, Puzanov I, Kim KB, Ribas A, McArthur GA, Sosman JA, O'Dwyer PJ, Lee RJ, Grippo JF, Nolop K and Chapman PB: Inhibition of mutated, activated BRAF in metastatic melanoma. N Engl J Med 363: 809-819, 2010.

4. Sosman JA, Kim KB, Schuchter L, Gonzalez R, Pavlick AC, Weber JS, McArthur GA, Hutson TE, Moschos SJ, Flaherty KT, et al: Survival in BRAF V600-mutant advanced melanoma treated with vemurafenib. N Engl J Med 366: 707-714, 2012.

5. Obenauf AC, Zou Y, Ji AL, Vanharanta S, Shu W, Shi H, Kong X, Bosenberg MC, Wiesner T, Rosen N, et al: Therapy-induced tumour secretomes promote resistance and tumour progression. Nature 520: 368-372, 2015.

6. Luebker SA and Koepsell SA: Diverse mechanisms of BRAF inhibitor resistance in melanoma identified in clinical and preclinical studies. Front Oncol 9: 268, 2019.

7. Long GV, Stroyakovskiy D, Gogas H, Levchenko E, de Braud F, Larkin J, Garbe C, Jouary T, Hauschild A, Grob JJ, et al: Combined BRAF and MEK inhibition versus BRAF inhibition alone in melanoma. N Engl J Med 371: 1877-1888, 2014.

8. Long GV, Weber JS, Infante JR, Kim KB, Daud A, Gonzalez R, Sosman JA, Hamid O, Schuchter L, Cebon J, et al: Overall survival and durable responses in patients with BRAF V600-mutant metastatic melanoma receiving dabrafenib combined with trametinib. J Clin Oncol 34: 871-878, 2016.

9. Sugie S, Okamoto K, Rahman KW, Tanaka T, Kawai K, Yamahara $\mathbf{J}$ and Mori $\mathrm{H}$ : Inhibitory effects of plumbagin and juglone on azoxymethane-induced intestinal carcinogenesis in rats. Cancer Lett 127: 177-183, 1998.

10. Polonik SG, Prokof'eva NG, Agafonova IG and Uvarova NI: Antitumor and immunostimulating activity of 5-hydroxy-1, 4-naphthoquinone (juglone) $\mathrm{O}$-and $\mathrm{S}$-acetylglycosides. Pharm Chem J 37: 397-398, 2003.

11. Aithal KB, Kumar SM, Rao NB, Udupa N and Rao SB: Juglone, a naphthoquinone from walnut, exerts cytotoxic and genotoxic effects against cultured melanoma tumor cells. Cell Biol Int 33: $1039-1049,2009$.

12. Aithal KB, Kumar S, Rao BN, Udupa N and Rao SBS: Tumor growth inhibitory effect of juglone and its radiation sensitizing potential: In vivo and in vitro studies. Integr Cancer Ther 11 $68-80,2012$

13. Liu X, Chen Y, Zhang Y, Du J, Lv Y, Mo S, Liu Y, Ding F, Wu J and Li J: Juglone potentiates TRAIL-induced apoptosis in human melanoma cells via activating the ROS-p38-p53 pathway. Mol Med Rep 16: 9645-9651, 2017.

14. Xu HL, Yu XF, Qu SC, Qu XR, Jiang YF and Sui da Y: Juglone, from Juglans mandshruica Maxim, inhibits growth and induces apoptosis in human leukemia cell HL-60 through a reactive oxygen species-dependent mechanism. Food Chem Toxicol 50: 590-596, 2012

15. Corazao-Rozas $\mathrm{P}$, Guerreschi $\mathrm{P}$, Jendoubi $\mathrm{M}$, André $\mathrm{F}$, Jonneaux A, Scalbert C, Garçon G, Malet-Martino M, Balayssac S, Rocchi S, et al: Mitochondrial oxidative stress is the Achille's heel of melanoma cells resistant to Braf-mutant inhibitor. Oncotarget 4: 1986-1998, 2013.

16. Feng Z, Kochanek S, Close D, Wang L, Srinivasan A, Almehizia AA, Iyer P, Xie XQ, Johnston PA and Gold B: Design and activity of AP endonuclease-1 inhibitors. J Chem Biol 8: 79-93, 2015.

17. Liu F, Cao J, Wu J, Sullivan K, Shen J, Ryu B, Xu Z, Wei W and Cui R: Stat3-targeted therapies overcome the acquired resistance to vemurafenib in melanomas. J Invest Dermatol 133: 2041-2049, 2013.

18. Guerriero L, Palmieri G, De Marco M, Cossu A, Remondelli P, Capunzo M, Turco MC and Rosati A: The anti-apoptotic BAG3 protein is involved in BRAF inhibitor resistance in melanoma cells. Oncotarget 8: 80393-80404, 2017.

19. Liu L, Li T, Tan J, Fu J, Guo Q, Ji H and Zhang Y: NG as a novel nitric oxide donor induces apoptosis by increasing reactive oxygen species and inhibiting mitochondrial function in MGC803 cells. Int Immunopharmacol 23: 27-36, 2014.
20. Chou TC: Drug combination studies and their synergy quantification using the Chou-Talalay method. Cancer Res 70: 440-446, 2010.

21. Soldani C and Scovassi AI: Poly(ADP-ribose) polymerase-1 cleavage during apoptosis: An update. Apoptosis 7: 321-328, 2002.

22. Ambrosini G, Adida C, Sirugo G and Altieri DC: Induction of apoptosis and inhibition of cell proliferation by survivin gene targeting. J Biol Chem 273: 11177-11182, 1998.

23. Shin S, Sung BJ, Cho YS, Kim HJ, Ha NC, Hwang JI, Chung CW, Jung $\mathrm{YK}$ and $\mathrm{Oh} \mathrm{BH}$ : An anti-apoptotic protein human survivin is a direct inhibitor of caspase-3 and-7. Biochemistry 40: 1117-1123, 2001.

24. Tang C, Liang J, Qian J, Jin L, Du M, Li M and Li D: Opposing role of JNK-p38 kinase and ERK1/2 in hydrogen peroxide-induced oxidative damage of human trophoblast-like JEG-3 cells. Int J Clin Exp Pathol 7: 959-968, 2014.

25. Renault TT and Manon S: Bax: Addressed to kill. Biochimie 93 1379-1391, 2011

26. Antonsson B, Conti F, Ciavatta A, Montessuit S, Lewis S, Martinou I, Bernasconi L, Bernard A, Mermod JJ, Mazzei G, et al: Inhibition of Bax channel-forming activity by Bcl-2. Science 277: 370-372, 1997.

27. Simon HU, Haj-Yehia A and Levi-Schaffer F: Role of reactive oxygen species (ROS) in apoptosis induction. Apoptosis 5: 415-418, 2000

28. Perfettini JL, Castedo M, Nardacci R, Ciccosanti F, Boya P, Roumier T, Larochette N,Piacentini M and Kroemer G: Essential role of p53 phosphorylation by $\mathrm{p} 38$ MAPK in apoptosis induction by the HIV-1 envelope. J Exp Med 201: 279-289, 2005.

29. Wu GS: The functional interactions between the MAPK and p53 signaling pathways. Cancer Biol Ther 3: 156-161, 2004.

30. Ji YB, Qu ZY and Zou X: Juglone-induced apoptosis in human gastric cancer SGC-7901 cells via the mitochondrial pathway. Exp Toxicol Pathol 63: 69-78, 2011.

31. Segura-Aguilar J, Jönsson K, Tidefelt U and Paul C: The cytotoxic effects of 5-OH-1,4-naphthoquinone and 5,8-diOH-1,4-naphthoquinone on doxorubicin-resistant human leukemia cells (HL-60). Leuk Res 16: 631-637, 1992.

32. Sajadimajd S and Yazdanparast R: Sensitizing effect of juglone is mediated by down regulation of Notch1 signaling pathway in trastuzumab-resistant SKBR3 cells. Apoptosis 22: 135-144, 2017.

33. Elmore S: Apoptosis: A review of programmed cell death. Toxicol Pathol 35: 495-516, 2007.

34. Xu HL, Yu XF, Qu SC, Zhang R, Qu XR, Chen YP, Ma XY and Sui DY: Anti-proliferative effect of Juglone from Juglans mandshurica Maxim on human leukemia cell HL- 60 by inducing apoptosis through the mitochondria-dependent pathway. Eur J Pharmacol 645: 14-22, 2010.

35. Ahmad T and Suzuki YJ: Juglone in oxidative stress and cell signaling. Antioxidants (Basel) 8: pii: E91, 2019.

36. Sidlauskas K, Sidlauskiene R, Li N and Liobikas J: 5-Hydroxy-1,4-naphthalenedione exerts anticancer effects on glioma cells through interaction with the mitochondrial electron transport chain. Neurosci Lett 639: 207-214, 2017.

37. Ziech D, Franco R, Pappa A and Panayiotidis MI: Reactive oxygen species (ROS)-induced genetic and epigenetic alterations in human carcinogenesis. Mutat Res 711: 167-173, 2011.

38. Martindale JL and Holbrook NJ: Cellular response to oxidative stress: Signaling for suicide and survival. J Cell Physiol 192: $1-15,2002$

39. Smeenk L, Van Heeringen SJ, Koeppel M, Gilbert B, Janssen-Megens E, Stunnenberg HG and Lohrum M: Role of p53 serine 46 in 553 target gene regulation. PLoS One 6: e17574, 2011.

40. Levine AJ and Oren M: The first 30 years of p53: Growing ever more complex. Nat Rev Cancer 9: 749-758, 2009.

41. Lu Z, Chen H, Zheng XM and Chen ML: Experimental study on the apoptosis of cervical cancer Hela cells induced by juglone through c-Jun N-terminal kinase/c-Jun pathway. Asian Pac J Trop Med 10: 572-575, 2017.

42. Graziani G, Artuso S, De Luca A, Muzi A, Rotili D, Scimeca M, Atzori MG, Ceci C, Mai A, Leonetti C, et al: A new water soluble MAPK activator exerts antitumor activity in melanoma cells resistant to the BRAF inhibitor vemurafenib. Biochem Pharmacol 95: 16-27, 2015.

43. Montenegro RC, Araújo AJ, Molina MT, Marinho Filho JD, Rocha DD, Lopéz-Montero E, Goulart MO, Bento ES, Alves AP, Pessoa C, et al: Cytotoxic activity of naphthoquinones with special emphasis on juglone and its 5-O-methyl derivative. Chem Biol Interact 184: 439-448, 2010 